\title{
Age and sex effects on advanced white matter microstructure measures in 15,628 older adults: A UK biobank study
}

\author{
Katherine E. Lawrence ${ }^{1}$. Leila Nabulsi ${ }^{1}$. Vigneshwaran Santhalingam ${ }^{1} \cdot$ Zvart Abaryan $^{1} \cdot$ Julio E. Villalon-Reina ${ }^{1}$. \\ Talia M. Nir ${ }^{1}$. Iyad Ba Gari ${ }^{1}$ • Alyssa H. Zhu ${ }^{1}$ - Elizabeth Haddad ${ }^{1}$. Alexandra M. Muir ${ }^{1}$. Emily Laltoo ${ }^{1}$. \\ Neda Jahanshad ${ }^{1} \cdot$ Paul M. Thompson ${ }^{1}$
}

Accepted: 23 August 2021 / Published online: 18 September 2021

(c) The Author(s) 2021

\begin{abstract}
A comprehensive characterization of the brain's white matter is critical for improving our understanding of healthy and diseased aging. Here we used diffusion-weighted magnetic resonance imaging (dMRI) to estimate age and sex effects on white matter microstructure in a cross-sectional sample of 15,628 adults aged $45-80$ years old ( $47.6 \%$ male, $52.4 \%$ female). Microstructure was assessed using the following four models: a conventional single-shell model, diffusion tensor imaging (DTI); a more advanced single-shell model, the tensor distribution function (TDF); an advanced multi-shell model, neurite orientation dispersion and density imaging (NODDI); and another advanced multi-shell model, mean apparent propagator MRI (MAPMRI). Age was modeled using a data-driven statistical approach, and normative centile curves were created to provide sex-stratified white matter reference charts. Participant age and sex substantially impacted many aspects of white matter microstructure across the brain, with the advanced dMRI models TDF and NODDI detecting such effects the most sensitively. These findings and the normative reference curves provide an important foundation for the study of healthy and diseased brain aging.
\end{abstract}

Keywords Aging $\cdot$ Sex differences $\cdot$ White matter $\cdot$ Diffusion-weighted MRI $\cdot$ Microstructure

\section{Introduction}

White matter alterations have been linked to age-related cognitive decline and implicated in neurodegenerative diseases such as Alzheimer's disease (Bennett \& Madden, 2014; Pievani et al., 2014). A range of age-associated neurodegenerative diseases also exhibit sex differences in their prevalence and presentation and, similar to age, sex has also been associated with white matter differences (Cox et al., 2016; Jahanshad \& Thompson, 2017; Ritchie et al., 2018; Salminen et al., 2020; Toschi et al., 2020). Understanding the brain's white matter may substantially improve our understanding of aging and sex differences therein, including ultimately the genetic and environmental factors that influence healthy or diseased aging. Diffusion-weighted magnetic

Paul M. Thompson

pthomp@usc.edu

1 Imaging Genetics Center, Mark and Mary Stevens Neuroimaging \& Informatics Institute, University of Southern California, Marina del Rey, CA, USA resonance imaging (dMRI) allows for the characterization of white matter microstructure by assessing the diffusion of water molecules in brain tissue (Stejskal \& Tanner, 1965). The conventional modeling approach applied to dMRI data, known as diffusion tensor imaging (DTI), fits a single-tensor to single-shell dMRI data and typically reflects hindered diffusion (Basser et al., 1994; Jones, 2008). A more advanced single-shell model is the tensor distribution function (TDF), which addresses well-established limitations of DTI by using a continuous mixture of tensors to capture multiple underlying fiber populations (Leow et al., 2009; Nir et al., 2017; Zhan et al., 2009). Compared to single-shell models, multi-shell dMRI models may allow for a more nuanced depiction of the underlying microstructural environment by using multi-shell dMRI data, which allows both hindered and restricted diffusion to be captured. Multi-shell diffusion models include, among others, the biophysical model neurite orientation dispersion and density imaging (NODDI) and the signal-based model mean apparent propagator MRI (MAPMRI). NODDI is a multi-compartment model that separately models restricted, hindered, and free water 
diffusion, which are thought to correspond to intra-cellular, extra-cellular, and isotropic water components, respectively (Zhang et al., 2012); NODDI may thus provide microstructure metrics more closely linked to specific aspects of the cellular environment than single-shell models (Zhang et al., 2012), although some recent work suggests the assumptions underlying NODDI's specificity may not always be met (Jelescu \& Budde, 2017; Jelescu et al., 2020). MAPMRI is a diffusion propagator-based multi-shell model that estimates the diffusion patterns of water molecules without a priori assumptions about the underlying tissue, which may allow for the detection of more subtle microstructure alterations (Fick et al., 2016; Le et al., 2020; Ning et al., 2015; Ozarslan et al., 2013).

Previous dMRI studies examining age and sex effects have reported age-related white matter decline and significant sex differences in white matter microstructure (Beck et al., 2020; Cox et al., 2016; Damoiseaux, 2017; Jahanshad \& Thompson, 2017; Ritchie et al., 2018; Salminen et al., 2020; Toschi et al., 2020; Tseng et al., 2020; ZavaliangosPetropulu et al., 2019). In one of the largest studies to date that investigated age and sex associations with white matter microstructure in adults, Cox et al. (2016) examined two DTI metrics (fractional anisotropy, FA; mean diffusivity, MD) and three NODDI metrics (orientation dispersion, OD; intra-cellular volume fraction, ICVF; isotropic volume fraction, ISOVF) in 3,513 middle-aged and older subjects from the UK Biobank for a range of white matter tracts across the brain; age was modeled using a linear or quadratic fit, based on the best fit for each tract and metric. These analyses indicated widespread effects of age and sex on most tracts for the examined DTI and NODDI metrics; interactions between age and sex exhibited small effect sizes and attained statistical significance for a limited subset of the tracts and metrics examined (Cox et al., 2016). A larger follow-up investigation in 5,216 UK Biobank participants assessed sex differences in one DTI metric (FA) and one NODDI metric (OD), among other non-dMRI measures, and similarly supported the existence of significant white matter differences between men and women for a range of tracts (Ritchie et al., 2018). In another study among 7,167 UK Biobank participants, Tseng et al. (2020) examined age effects on white matter using a linear age model and four microstructure metrics analogous to FA or calculated using DTI (generalized fractional anisotropy, GFA; mean diffusivity, MD; axial diffusivity, AD; radial diffusivity, RD). Their results further supported the association between age and white matter decline, in addition to indicating a sex difference in the overall number of white matter tracts that exhibited age-related declines in anisotropy (Tseng et al., 2020). Such prior dMRI work robustly demonstrates that multiple aspects of white matter microstructure are significantly associated with participant age and sex (Beck et al., 2020; Cox et al., 2016; Damoiseaux, 2017; Jahanshad \& Thompson, 2017; Ritchie et al., 2018; Salminen et al., 2020; Toschi et al., 2020; Tseng et al., 2020; Zavaliangos-Petropulu et al., 2019). However, it remains an open question how age, sex, and their interaction may be related to additional measures of white matter microstructure obtained from other advanced dMRI models such as TDF and MAPMRI, as well as how age effects on microstructure may manifest in middle to late adulthood when using more complex, data-driven statistical approaches for modeling age. Notably, nearly all dMRI studies to date focusing on aging or sex differences have used only one or two dMRI models to investigate white matter, but using multiple dMRI models in a single study may allow for a more complete characterization of white matter microstructure, including which white matter measures may be most sensitive to age and sex (Cox et al., 2016; Ritchie et al., 2018; Toschi et al., 2020; Tseng et al., 2020; ZavaliangosPetropulu et al., 2019). Prior large-scale dMRI analyses have also assumed that white matter aging trajectories are linear or quadratic with respect to age, but aging trajectories may be captured more accurately by data-driven statistical techniques (Cox et al., 2016; Dima et al., 2020; Frangou et al., 2020; Pietrasik et al., 2020; Tseng et al., 2020).

Our understanding of aging would also benefit from establishing typical ranges of white matter properties among middle-aged and older adults. Such normative reference data would allow future investigations to detect individuals with quantifiably abnormal white matter microstructure for their age and sex, such as individuals who are below the $5^{\text {th }}$ percentile or above the $95^{\text {th }}$ percentile for a given microstructure metric (Marquand et al., 2019; Wolfers et al., 2018; Zabihi et al., 2019). As many age-related neurodegenerative diseases are associated with altered white matter, such normative white matter reference curves may allow future aging work to identify individuals with the most severe neural pathology (Pievani et al., 2014). This would provide a novel avenue for future pre-clinical aging studies by allowing them to characterize those factors associated with the most diseased neural phenotypes. Prior large-scale neuroimaging has computed normative reference values for a range of structural morphometry measures, such as hippocampal volume, as well as several microstructure metrics (Dima et al., 2020; Frangou et al., 2020; Nobis et al., 2019; Pomponio et al., 2020; Tseng et al., 2020). Normative reference curves for additional white matter microstructural properties would provide complementary information and, together with preexisting neural reference values, allow for a more complete characterization of healthy and diseased aging in future studies.

Here we expanded on prior work by using multiple dMRI models to thoroughly characterize age and sex effects on white matter microstructure in a large-scale, populationbased sample of middle-aged and older adults. Specifically, 
we examined age and sex associations with DTI, TDF, NODDI, and MAPMRI microstructure metrics in 15,628 cross-sectional UK Biobank participants in what is, to the best of our knowledge, the largest such published study to date. Age was modeled non-linearly using a data-driven statistical approach, and normative centile curves were calculated for all dMRI measures to provide sex-stratified references for white matter. We found that age and participant sex was significantly related to many white matter properties across the brain, and advanced dMRI models detected age and sex effects the most sensitively. The computed reference curves provide a novel avenue for future studies focused on the characterization of white matter in middle to late adulthood.

\section{Methods}

\section{Study design, MRI acquisition and processing}

We analyzed cross-sectional dMRI data from a total of 15,628 community-based UK Biobank subjects aged 45-80 years (47.6\% male; Table S1) (Miller et al., 2016). All dMRI data included in the current study was collected on a single scanner. Sample details and dMRI processing are presented in the Supplementary Material (Supplementary Methods). Briefly, white matter metrics were derived using four dMRI reconstruction models: DTI, TDF, NODDI, and MAPMRI. Each reconstruction method is further described in the Supplementary Material (Supplementary Methods). Metrics derived from DTI included fractional anisotropy $\left(\mathrm{FA}^{\mathrm{DTI}}\right)$, mean diffusivity (MD), axial diffusivity (AD), and radial diffusivity (RD). An advanced measure of fractional anisotropy was calculated using TDF (FA $\left.{ }^{\mathrm{TDF}}\right)$. Measures derived from NODDI included orientation dispersion (OD), intra-cellular volume fraction (ICVF), and isotropic volume fraction (ISOVF). The following white matter indices were calculated from MAPMRI: return-to-origin probability (RTOP), return-to-axis probability (RTAP), and return-toplane probability (RTPP); RTOP is a zero-displacement probability that can be decomposed into RTAP and RTPP, which reflect restrictive barriers in the radial and axial orientations, respectively. Diffusion-weighted MRI metrics were projected to a standard white matter skeleton using publicly available ENIGMA protocols based on FSL's tract-based statistics (TBSS) and described further in the Supplementary Material (Supplementary Methods) (http://enigma.ini.usc. edu/protocols/dti-protocols; Jahanshad et al., 2013; Smith et al., 2006). Consistent with prior literature, we focused on mean microstructure values for the whole white matter skeleton (full WM) and the corpus callosum (CC) (e.g., Beck et al., 2020; Jahanshad \& Thompson, 2017; Pines et al., 2020). For completeness, supplemental analyses examined mean values in additional atlas-based white matter regions of interest (ROIs) across the brain (Supplemental Methods).

\section{Statistical analyses}

Our planned a priori analyses investigated the effects of age, sex, and their interaction on the full WM and CC by using fractional polynomials to flexibly model age in a non-linear manner (Dima et al., 2020; Frangou et al., 2020; Royston \& Altman, 1994). The fractional polynomial approach is detailed further in the Supplementary Material (Supplementary Methods), as are the nuisance covariates we included based on prior literature (Salminen et al., 2019). Effect sizes were calculated as the variance explained separately by age, sex, and their interaction. For instance, the effect size for age was computed as the difference in variance (change in $\mathrm{R}^{2}$ ) between two models: one which included age in addition to sex and nuisance covariates, and one which only included sex and nuisance covariates. The overall $\mathrm{R}^{2}$ for each full model is reported in the Supplementary Material. Sex-stratified centile reference curves were created for each atlas-based white matter ROI and dMRI metric using quantile regression (Supplementary Methods). For completeness, supplemental analyses used stricter statistical thresholds ( $p<0.01$ and $p<0.001)$, in addition to examining age and sex effects on an additional 14 atlas-based white matter ROIs across the brain (Table S2) and using a distinct statistical approach to model age; details on these supplemental analyses are presented in the Supplementary Material (Supplementary Methods).

\section{Results}

Age was robustly associated with the full WM and $\mathrm{CC}$ for all dMRI metrics (Fig. 1A-B, Fig. 2, Fig. 3, Fig. S24-S45, Fig. S46-S67). Such age effects in our cross-sectional sample were observed in the full $\mathrm{WM}$ and $\mathrm{CC}$ as lower anisotropy $\left(\mathrm{FA}^{\mathrm{DTI}}, \mathrm{FA}^{\mathrm{TDF}}\right)$, neurite density (ICVF), and restriction (RTOP, RTAP, RTPP), as well as higher diffusivity (MD, $\mathrm{AD}, \mathrm{RD}$ ) and free water (ISOVF) with increasing age. White matter dispersion (OD) was higher in the full WM and lower in the CC with increasing age. Follow-up analyses using more stringent statistical thresholds yielded identical results (Fig. S1A-B, Fig. S2A-B). Effect sizes for age in the full WM and $C C$ demonstrated that the advanced single-shell model, TDF, was most sensitive to age, followed by DTI and NODDI (Fig. 1A-B). Supplemental analyses examining additional atlas-based white matter ROIs across the brain and using a distinct statistical approach to model age likewise demonstrated that TDF was the most sensitive dMRI model, followed by DTI (Fig. S3A, Fig. S4A, Fig. S5A, Fig. S6-S19, Fig. S20A-B, Fig. S21A, Fig. S68-S375). Total 
(A) Age: Full White Matter

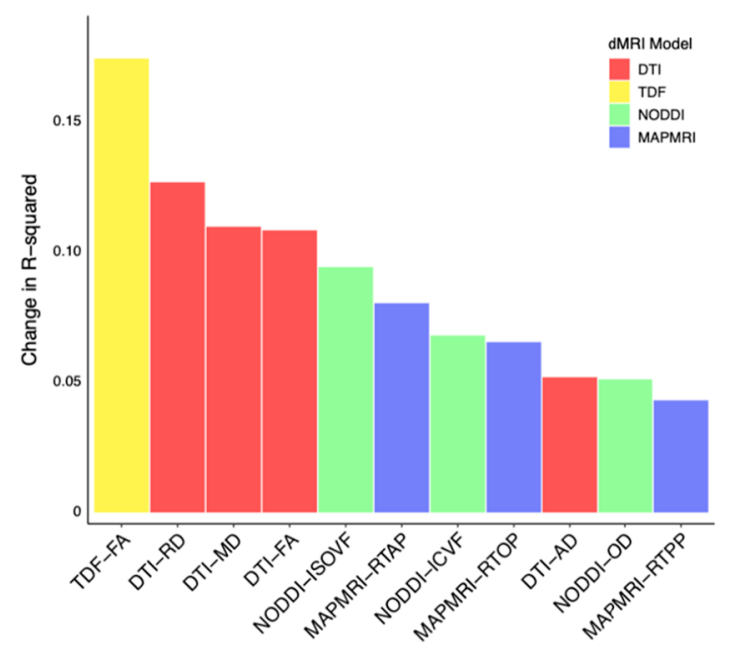

(C) Sex: Full White Matter

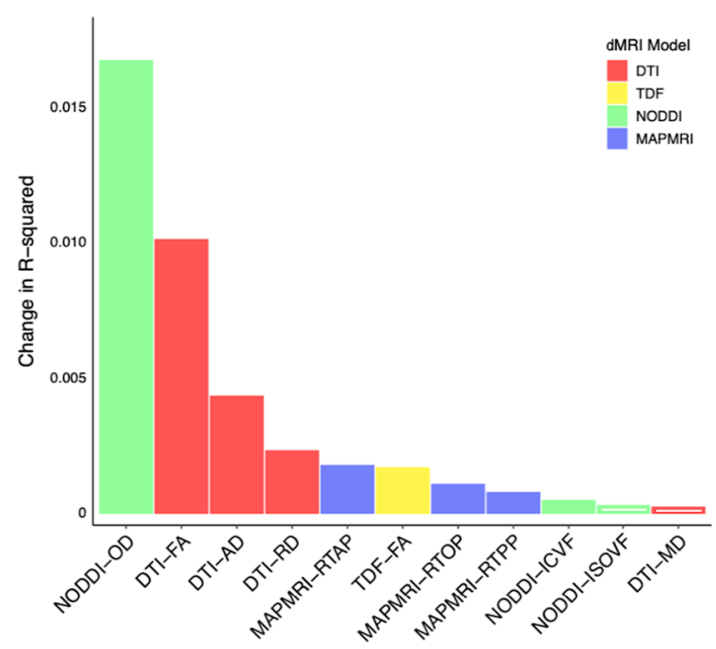

(E) Age $\times$ Sex: Full White Matter

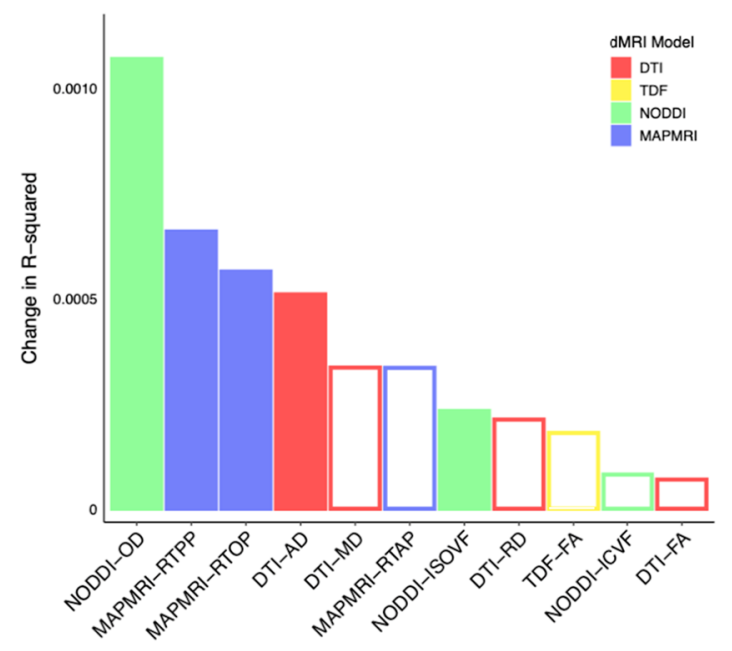

(B) Age: Corpus Callosum

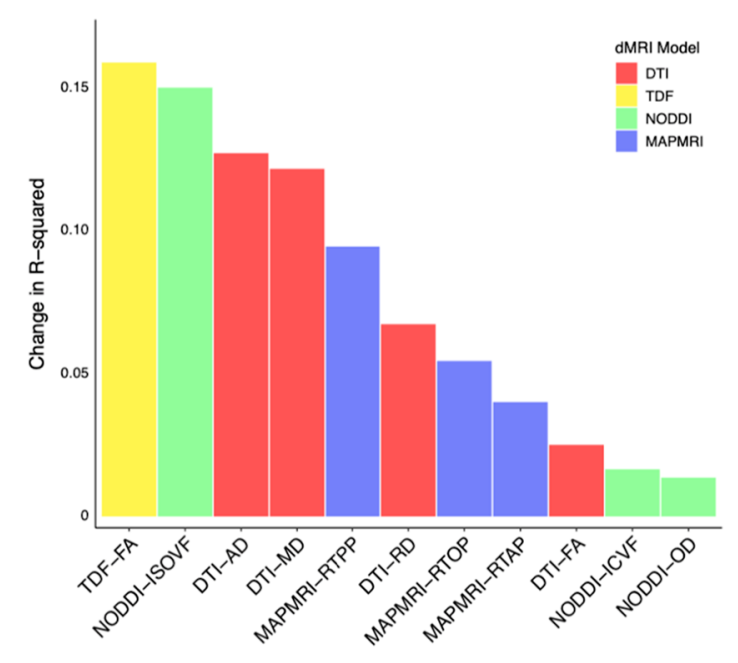

(D) Sex: Corpus Callosum

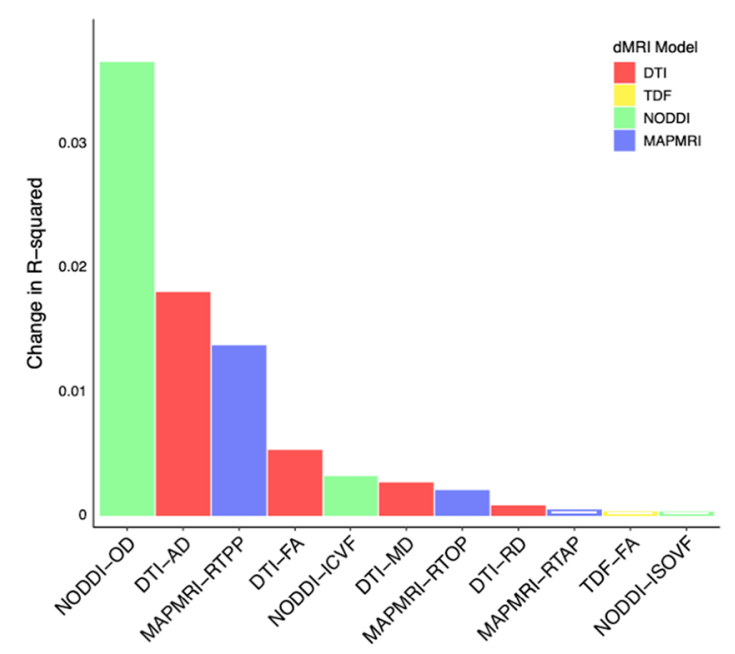

(F) Age $\times$ Sex: Corpus Callosum

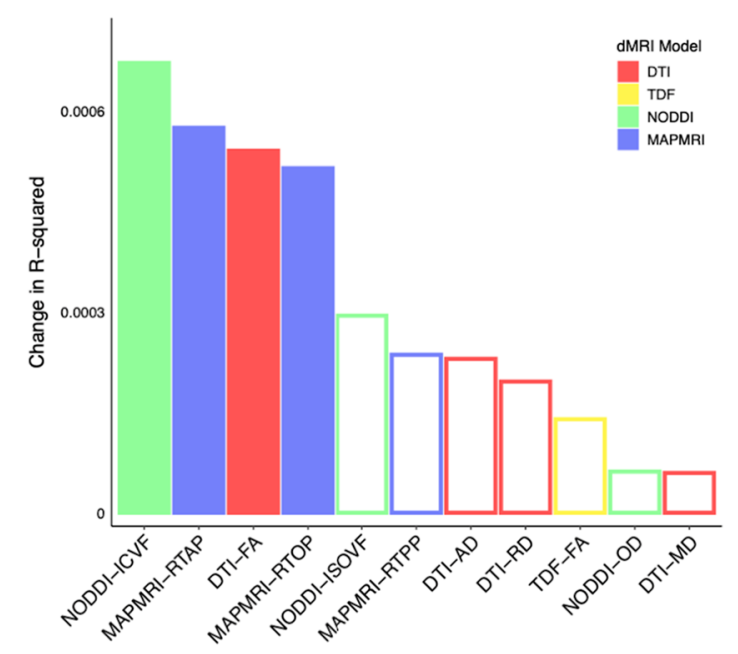


४Fig. 1 Effect of age (A-B), participant sex (C-D), and their interaction $(\mathrm{E}-\mathrm{F})$ on full white matter and corpus callosum microstructure. Age was modeled as a continuous variable using fractional polynomials. Filled bars indicate a significant association $(p<0.05)$, whereas hollow bars indicate the association did not attain statistical significance

variance explained by the full models including age, sex, their interaction, and covariates of non-interest are reported in the Supplementary Material (Fig. S22, Fig. S23). In sum, age has widespread effects on white matter microstructure that are detected most sensitively by TDF.

Participant sex was significantly associated with full WM and CC microstructure for nearly all assessed dMRI indices (Fig. 1C-D, Fig. 2, Fig. 3, Fig. S24-S45, Fig. S46-S67). Patterns of sex differences were typically region- and metricspecific. In both the full WM and the CC, women exhibited greater RD, OD, and RTPP than men; men displayed greater $\mathrm{FA}^{\mathrm{DTI}}$ and $\mathrm{AD}$ compared with women. For full WM, male participants also displayed higher FA ${ }^{\mathrm{TDF}}$, ICVF, RTOP, and RTAP. When contrasting CC microstructure between men and women, women had greater ICVF and RTOP. Men exhibited higher MD in the CC than women. Results were highly similar when applying stricter statistical thresholds, except sex differences no longer attained significance in the full WM for ICVF $(p<0.01)$ and RTPP $(p<0.001)$, as well as in the CC for RD $(p<0.01)$ (Fig. S1C-D, Fig. S2CD). Effect sizes for full WM and CC revealed that sex differences were detected most sensitively by the advanced multi-shell model, NODDI, followed by DTI (Fig. 1C-D). Follow-up analyses investigating supplemental atlas-based white matter ROIs and using different methods to model age similarly found that NODDI and DTI were most sensitive to sex differences (Fig. S3B, Fig. S4B, Fig. S5B, Fig. S6-S19, Fig. S20C-D, Fig. S21B, Fig. S68-S375). Effect sizes for the complete models including age, sex, their interaction and covariates are included in the Supplementary Material (Fig. S22, Fig. S23). Together, these results indicate that participant sex is robustly associated with white matter across the brain, and such sex differences are captured most sensitively by NODDI.

Age effects on the full WM and CC significantly depended on participant sex for multiple dMRI metrics (Fig. 1E-F, Fig. 2, Fig. 3, Fig. S24-S45, Fig. S46-S67). These age by sex interactions in our cross-sectional sample were driven by complex combinations of steeper age-related decline among women than men and vice versa. The most common pattern was generally steeper decline in female than male participants, as observed in both the full WM (AD, ISOVF, RTPP) and the CC (ICVF, RTOP). A mixture of faster, slower, and similar age-associated decline in female compared to male subjects was also observed to a somewhat lesser extent, in both full WM (RTOP) and the CC (FA ${ }^{\text {DTI }}$, RTAP). In full
WM only, men displayed steeper age-related decline than women in fiber dispersion (OD). Follow-up analyses using a stricter statistical threshold $(p<0.01)$ yielded a largely similar pattern of results except the interaction between age and sex was no longer significant in the full $\mathrm{WM}$ for $\mathrm{AD}$ and in the CC for FA ${ }^{\text {DTI }}$ and RTOP (Fig. S1E-F). When using an even more stringent threshold $(p<0.001)$, sex differences in aging no longer attained statistical significance for full WM measures of RTPP and RTOP, as well as CC measures of ICVF and RTAP (Fig. S2E-F). Effect sizes for the age by sex interaction in the full WM and CC indicated that the multi-shell model, NODDI, was most sensitive to such sex differences in age effects, followed by MAPMRI and DTI (Fig. 1E-F). Supplementary analyses characterizing further atlas-based white matter ROIs, and testing the robustness of results to the selected age model, similarly indicated that NODDI and DTI exhibited the greatest effect sizes (Fig. S3C, Fig. S4C, Fig. S5C, Fig. S6-S19, Fig. S20EF, Fig. S21C, Fig. S68-S375). Effect sizes for the full models are presented in the Supplementary Material (Fig. S22, Fig. S23). As a whole, the effect of age significantly depends on participant sex for many white matter ROIs and measures, and NODDI detects such sex differences in age effects the most sensitively.

To provide normative models of white matter microstructure for the full WM and CC, sex-stratified centile reference curves were calculated for each dMRI metric (Fig. 2, Fig. 3, Fig. S24-S45, Fig. S46-S67). For completeness, normative reference charts for an additional 14 atlas-based white matter ROIs across the brain are included in the Supplementary Material (Fig. S2-S15, Fig. S68-S375).

\section{Discussion}

Here we thoroughly characterized age and sex effects on white matter microstructure by using an array of dMRI models coupled with advanced statistical methods. We also created normative reference curves for multiple aspects of white matter micro-architecture, which may allow for the future identification of individuals with the greatest neural pathology. As a whole, age and participant sex was robustly related to white matter across the brain, with the advanced dMRI models TDF and NODDI capturing such differences the most sensitively.

Age was associated with white matter alterations in nearly every region and microstructural property investigated. Specifically, older age in our cross-sectional sample was related to mostly lower anisotropy, neurite density, and restriction, as well as mostly higher diffusivity and free water; agedependent changes in fiber dispersion were more regionally specific. These results are in line with postmortem histological findings that aging is associated with the degradation and 

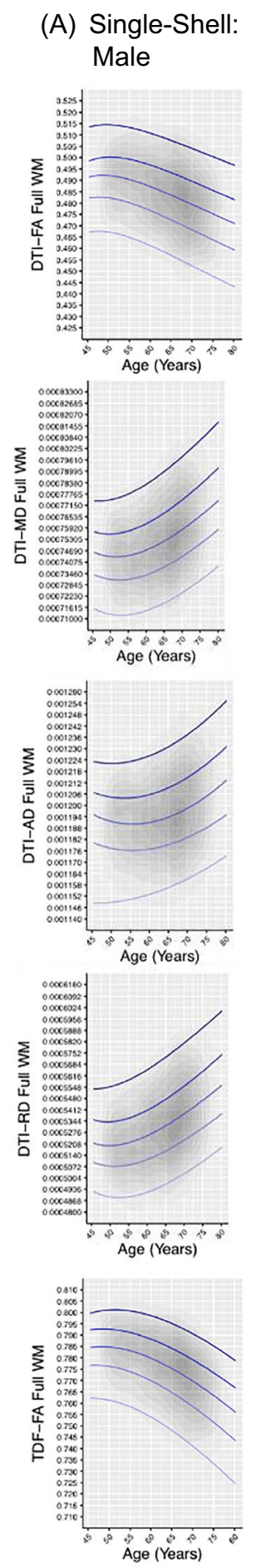

(B) Single-Shell:
Female
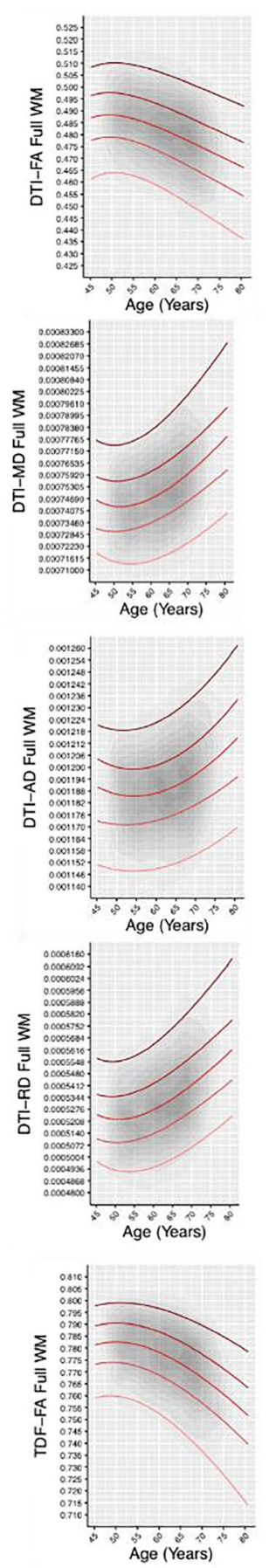

(C) Multi-Shell:

Male
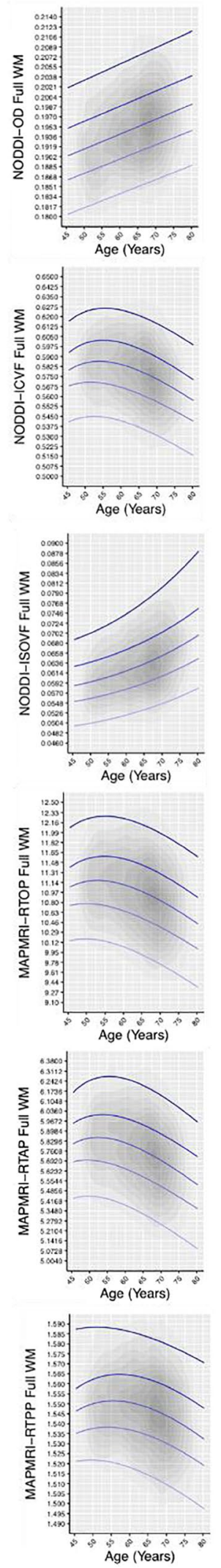

(D) Multi-Shell:

\section{Female}
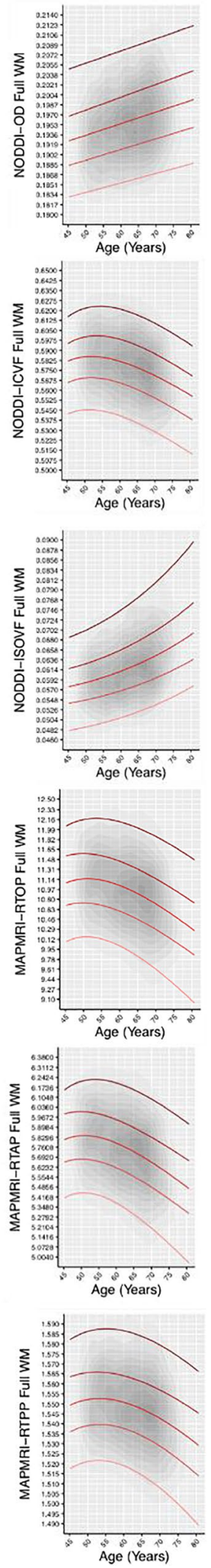
4Fig. 2 Normative centile reference curves calculated for the full white matter for single-shell dMRI metrics in (A) males and (B) females, and multi-shell dMRI metrics in (C) males and (D) females. Solid colored lines, ordered from lightest to darkest, indicate the following centiles: $5^{\text {th }}, 25^{\text {th }}, 50^{\text {th }}, 75^{\text {th }}, 95^{\text {th }}$; blue lines indicate male participants, and red lines indicate female participants. Gray overlay reflects kernel density (darker $=$ greater degree of data point overlap). Full $\mathrm{WM}=$ full white matter skeleton

deformation of axons and myelination (Bennett \& Madden, 2014). Our age results are also consistent with prior dMRI work that examined one or two dMRI models among the DTI, TDF, NODDI, and MAPMRI approaches included here (Beck et al., 2020; Bennett \& Madden, 2014; Cox et al., 2016; Damoiseaux, 2017; Tseng et al., 2020; ZavaliangosPetropulu et al., 2019). Notably, white matter microstructure in aging has previously been linked to age-related cognitive decline and neurodegenerative diseases, underscoring the importance of understanding age effects on white matter among older adults (Bennett \& Madden, 2014; Pievani et al., 2014).

Participant sex was also significantly related to many white matter properties across the brain although, notably, the magnitude of sex effects was substantially smaller than that of age effects. As a whole, women exhibited greater white matter dispersion than men, on average. Men displayed greater anisotropy, on average, compared to women. Sex differences in other white matter microstructure characteristics - including measures reflecting diffusivity, neurite density, free water, and restriction - depended to a greater extent on the exact region and metric examined. These findings are largely consistent with previous analyses which used DTI or NODDI to assess white matter sex difference (Cox et al., 2016; Jahanshad \& Thompson, 2017; Ritchie et al., 2018; Salminen et al., 2020; Toschi et al., 2020); such prior analyses likewise found that males exhibit higher anisotropy and lower fiber dispersion than females, with other microstructure metrics displaying greater variability in their pattern of sex differences. In the current study, we also observed significant sex differences in age effects for a number of regions and microstructure metrics in our cross-sectional sample; the magnitude of these interactions between age and sex was substantially smaller than the magnitude of the main effects of age or sex. The most common pattern for the interaction between age and sex was steeper age-related decline among women than men. Previous dMRI work has likewise suggested sex differences in age effects (Cox et al., 2016; Kodiweera et al., 2016; Toschi et al., 2020; Tseng et al., 2020). The largest of these studies investigated 7,167 UK Biobank participants and found that, overall, a greater number of tracts exhibited age-related declines in anisotropy among women than men (Tseng et al., 2020). Another study in 3,513 subjects from the UK Biobank demonstrated that age effects significantly differed between men and women for a small number of tracts and microstructure metrics, where the directionality of such differences depended on the specific tract and metric (Cox et al., 2016). Our findings expand on this prior work indicating sex differences in age effects among middle-aged to older adults. Importantly, a range of neurodegenerative conditions exhibit sex differences in their prevalence and presentation, emphasizing the importance of understanding differences in neural aging between men and women (Salminen et al., 2020).

Advanced dMRI models detected age and sex effects most sensitively in the current study. We used four separate dMRI models to characterize white matter microstructure - DTI, TDF, NODDI, and MAPMRI - and found that the advanced models TDF and NODDI exhibited the greatest sensitivity to white matter differences. Notably, between the two single-shell models DTI and TDF, TDF may be considered to model the underlying neurobiology more directly than DTI; among the two multi-shell models, NODDI may similarly be considered to model the underlying biology more directly than MAPMRI. More specifically, among the singleshell dMRI approaches, TDF models multiple underlying fibers per voxel, whereas DTI cannot differentiate multiple fiber populations (Jones, 2008; Leow et al., 2009; Nir et al., 2017; Zhan et al., 2009). Similarly, among the multi-shell dMRI models included in the current study, NODDI directly models multiple aspects of the cellular environment whereas MAPMRI estimates diffusion patterns without specifically modeling the underlying biology (Fick et al., 2016; Ozarslan et al., 2013; Zhang et al., 2012). This suggests that dMRI approaches which model the underlying neurobiology may capture microstructural differences more sensitively in community-based samples of middle-aged and older adults such as the UK Biobank sample examined here. As TDF was the most sensitive to age and NODDI to participant sex, our results furthermore indicate that the relative utility of each dMRI model depends not only on the fidelity of the model to the underlying neurobiology, but also on the specific neurobiology underlying the scientific question of interest. Future work should assess whether the relative utility of different dMRI models may depend on the dMRI analysis method used (e.g., TBSS vs. tractography-based approaches). As a whole, these findings indicate that future research examining age and sex may benefit from including advanced dMRI measures.

The current study has a number of important strengths, including our use of multiple dMRI models, our data-driven approach to model age, and the unprecedented sample size. The age and sex findings presented here may serve as a reference for future analyses investigating the genetic and environmental factors that contribute to healthy or diseased aging. The normative centile charts provided here may also allow for the detection of individuals with abnormal white matter, contributing to future studies focused on the 

(A) Single-Shell: Male
(B) Single-Shell:
Female
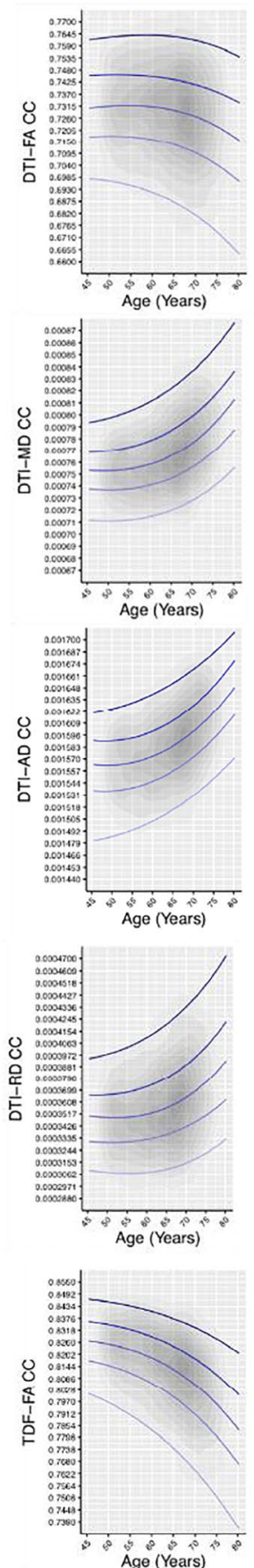
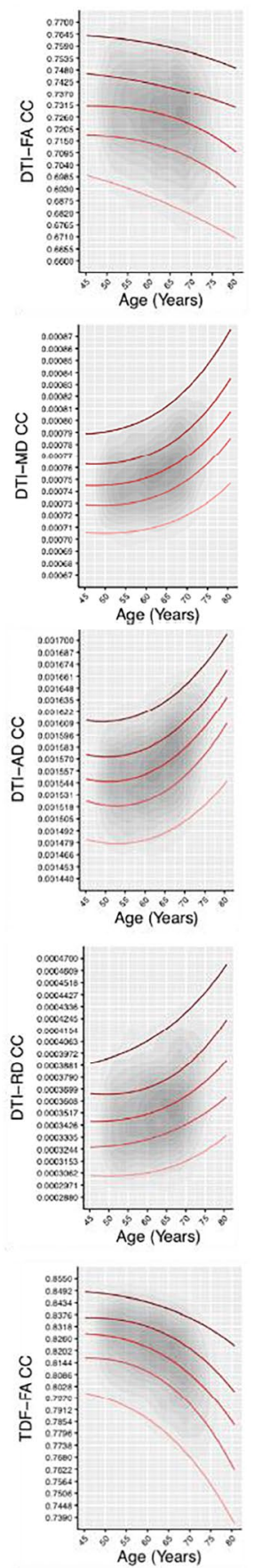

(C) Multi-Shell: Male
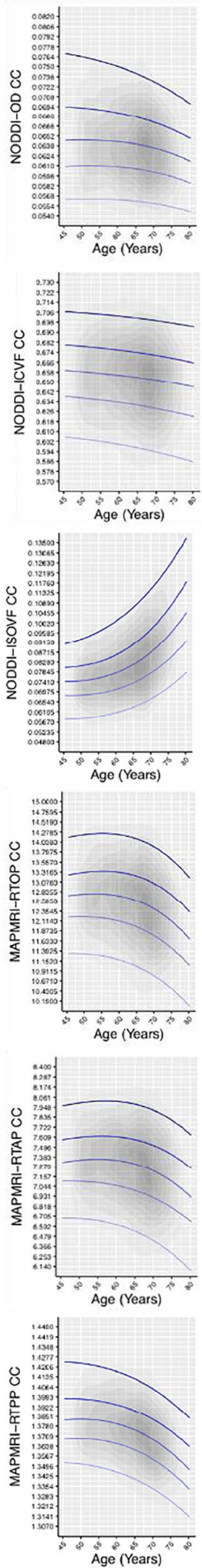

(D) Multi-Shell: Female
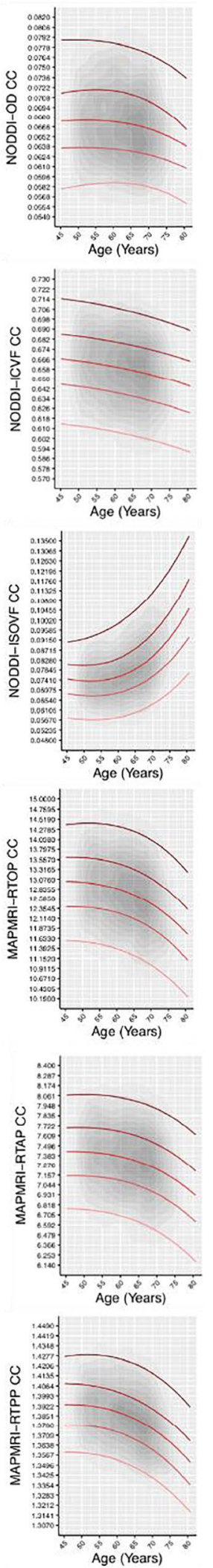
4Fig. 3 Normative centile reference curves calculated for the corpus callosum for single-shell dMRI metrics in (A) males and (B) females, and multi-shell dMRI metrics in (C) males and (D) females. Solid colored lines, ordered from lightest to darkest, indicate the following centiles: $5^{\text {th }}, 25^{\text {th }}, 50^{\text {th }}, 75^{\text {th }}, 95^{\text {th }}$; blue lines indicate male participants, and red lines indicate female participants. Gray overlay reflects kernel density (darker $=$ greater degree of data point overlap). $\mathrm{CC}=$ corpus callosum

characterization of diseased aging. Future work should expand on the current study by examining longitudinal samples and including additional dMRI analysis methods beyond the TBSS approach used here, such as tractographybased analyses. Future studies should further validate our findings in diverse healthy and diseased datasets collected on different scanners, as the UK Biobank is a community-based study and the sample used here was not selected based on the absence or presence of specific pathologies. Additionally, age was not uniformly distributed in the current sample and regression-based approaches, such as those used here, inherently exhibit greater variability in model estimates at the tails of the distribution. Future work including a wider age range with a uniform distribution will thus further improve the estimated model precision, particularly in the youngest (45 years) and oldest ( 80 years) participants. Future studies should also assess the generalizability of our normative reference curves across dMRI acquisition protocols that vary in the number and $b$-value of diffusion-weighted shells.

\section{Conclusions}

In summary, we characterized white matter microstructure during middle to late adulthood in 15,628 individuals using multiple dMRI models. Age and participant sex exhibited widespread associations with white matter across the brain, and advanced dMRI models demonstrated the greatest sensitivity to such effects. These findings provide an important foundation for the study of healthy and diseased aging.

\section{Funding sources}

This research was supported by the National Institute of Aging (award numbers R56AG058854 and U01 AG068057 to P.M.T., R01AG059874 to N.J., and T32AG058507 to T.M.N), the National Institute of Biomedical Imaging and Bioengineering (award number P41EB015922 to P.M.T.), the National Institute of Mental Health (award number F32MH122057 to K.E.L.), and a grant from Biogen, Inc. (to P.M.T. and N.J.). The contents of this paper are solely the responsibility of the authors and do not necessarily represent the views of the funders.
Supplementary Information The online version contains supplementary material available at https://doi.org/10.1007/s11682-021-00548-y.

Acknowledgements This research was conducted using the UK Biobank resource under Application 11559.

Author contributions Author contributions included conceptualization (K.E.L., L.N., N.J., P.M.T.), software (K.E.L., L.N., V.S., Z.A., J.E.V-R., T.M.N., I.B.G., A.H.Z., E.H.), formal analysis (K.E.L., L.N.), resources (N.J., P.M.T.), data curation (K.E.L., L.N., Z.A., A.H.Z., A.M.M., N.J.), visualization (K.E.L., E.L.), funding acquisition (N.J., P.M.T.), writing the original draft (K.E.L., N.J., P.M.T.), revising the draft for important intellectual content (all authors), and approving the final version for publication (all authors).

Availability of data and material Diffusion-weighted MRI data are available through the UK Biobank application procedure (https://www. ukbiobank.ac.uk/register-apply/).

Code availability Code for the tensor distribution protocol (TDF) is publicly available here: https://resource.loni.usc.edu/resources/downl oads/tensor-distribution-function-code/. Code for the ENIGMA diffusion-weighted MRI protocol is publicly available here: http://enigma. ini.usc.edu/protocols/dti-protocols.

\section{Declarations}

Ethical approval Not applicable.

Consent to participate Not applicable.

Consent to publish The manuscript has been approved by all coauthors. This material has not been published previously and has not been submitted elsewhere for publication.

Competing interests All authors declare no competing interests.

Open Access This article is licensed under a Creative Commons Attribution 4.0 International License, which permits use, sharing, adaptation, distribution and reproduction in any medium or format, as long as you give appropriate credit to the original author(s) and the source, provide a link to the Creative Commons licence, and indicate if changes were made. The images or other third party material in this article are included in the article's Creative Commons licence, unless indicated otherwise in a credit line to the material. If material is not included in the article's Creative Commons licence and your intended use is not permitted by statutory regulation or exceeds the permitted use, you will need to obtain permission directly from the copyright holder. To view a copy of this licence, visit http://creativecommons.org/licenses/by/4.0/.

\section{References}

Basser, P. J., Mattiello, J., \& Lebihan, D. (1994). Mr Diffusion Tensor Spectroscopy and Imaging. Biophysical Journal, 66(1), 259-267. https://doi.org/10.1016/S0006-3495(94)80775-1

Beck, D., de Lange, A. G., Maximov, I. I., Richard, G., Andreassen, O. A., Nordvik, J. E., \& Westlye, L. T. (2020). White matter microstructure across the adult lifespan: A mixed longitudinal and cross-sectional study using advanced diffusion models and 
brain-age prediction. NeuroImage, 224, 117441. https://doi.org/ 10.1016/j.neuroimage.2020.117441

Bennett, I. J., \& Madden, D. J. (2014). Disconnected aging: Cerebral white matter integrity and age-related differences in cognition. Neuroscience, 276, 187-205. https://doi.org/10.1016/j.neuroscien ce.2013.11.026

Cox, S. R., Ritchie, S. J., Tucker-Drob, E. M., Liewald, D. C., Hagenaars, S. P., Davies, G., ..., Deary, I. J. (2016). Ageing and brain white matter structure in 3,513 UK Biobank participants.Nature Communications7, 13629. https://doi.org/10.1038/ncomms13629

Damoiseaux, J. S. (2017). Effects of aging on functional and structural brain connectivity. NeuroImage, 160, 32-40. https://doi.org/10. 1016/j.neuroimage.2017.01.077

Dima, D., Papachristou, E., Modabbernia, A., Doucet, G. E., Agartz, I., Aghajani, M., ... Frangou, S. (2020). Subcortical Volume Trajectories across the Lifespan: Data from 18,605 healthy individuals aged 3-90 years. bioRxiv, 2020.2005.2005.079475. https://doi. org/10.1101/2020.05.05.079475

Fick, R. H. J., Wassermann, D., Caruyer, E., \& Deriche, R. (2016). MAPL: Tissue microstructure estimation using Laplacian-regularized MAP-MRI and its application to HCP data. NeuroImage, 134, 365-385. https://doi.org/10.1016/j.neuroimage.2016.03.046

Frangou, S., Modabbernia, A., Doucet, G. E., Papachristou, E., Williams, S. C., Agartz, I., . . Dima, D. (2020). Cortical Thickness Trajectories across the Lifespan: Data from 17,075 healthy individuals aged 3-90 years. bioRxiv, 2020.2005.2005.077834. https://doi.org/10.1101/2020.05.05.077834

Jahanshad, N., Kochunov, P. V., Sprooten, E., Mandl, R. C., Nichols, T. E., Almasy, L., ..., Glahn, D. C. (2013). Multi-site genetic analysis of diffusion images and voxelwise heritability analysis: a pilot project of the ENIGMA-DTI working group. Neuroimage, 81 , 455-469. https://doi.org/10.1016/j.neuroimage.2013.04.061

Jahanshad, N., \& Thompson, P. M. (2017). Multimodal neuroimaging of male and female brain structure in health and disease across the life span. Journal of Neuroscience Research, 95(1-2), 371-379. https://doi.org/10.1002/jnr.23919

Jelescu, I. O., Palombo, M., Bagnato, F., \& Schilling, K. G. (2020). Challenges for biophysical modeling of microstructure. Journal of Neuroscience Methods, 344, 108861. https://doi.org/10.1016/j. jneumeth.2020.108861

Jelescu, I. O., \& Budde, M. D. (2017). Design and validation of diffusion MRI models of white matter. Fronters of Physics, 28. https:// doi.org/10.3389/fphy.2017.00061

Jones, D. K. (2008). Studying connections in the living human brain with diffusion MRI. Cortex, 44(8), 936-952. https://doi.org/10. 1016/j.cortex.2008.05.002

Kodiweera, C., Alexander, A. L., Harezlak, J., McAllister, T. W., \& Wu, Y. C. (2016). Age effects and sex differences in human brain white matter of young to middle-aged adults: A DTI, NODDI, and q-space study. NeuroImage, 128, 180-192. https://doi.org/ 10.1016/j.neuroimage.2015.12.033

Le, H., Zeng, W., Zhang, H., Li, J., Wu, X., Xie, M., ..., Shen, J. (2020). Mean Apparent Propagator MRI Is Better Than Conventional Diffusion Tensor Imaging for the Evaluation of Parkinson's Disease: A Prospective Pilot Study. Front Aging Neurosci, 12, 563595. https://doi.org/10.3389/fnagi.2020.563595

Leow, A. D., Zhu, S., Zhan, L., McMahon, K., de Zubicaray, G. I., Meredith, M., ..., Thompson, P. M. (2009). The tensor distribution function. Magn Reson Med, 61(1), 205-214.https://doi.org/ $10.1002 / \mathrm{mrm} .21852$

Marquand, A. F., Kia, S. M., Zabihi, M., Wolfers, T., Buitelaar, J. K., \& Beckmann, C. F. (2019). Conceptualizing mental disorders as deviations from normative functioning. Molecular Psychiatry, 24(10), 1415-1424. https://doi.org/10.1038/s41380-019-0441-1

Miller, K. L., Alfaro-Almagro, F., Bangerter, N. K., Thomas, D. L., Yacoub, E., Xu, J., ..., Smith, S. M. (2016). Multimodal population brain imaging in the UK Biobank prospective epidemiological study. Nature Neuroscience, 19(11), 1523-1536. https://doi.org/ $10.1038 / \mathrm{nn} .4393$

Ning, L., Laun, F., Gur, Y., DiBella, E. V., Deslauriers-Gauthier, S., Megherbi, T., ..., Rathi, Y. (2015). Sparse Reconstruction Challenge for diffusion MRI: Validation on a physical phantom to determine which acquisition scheme and analysis method to use? Medical Image Analysis, 26(1), 316-331. https://doi.org/10.1016/j. media.2015.10.012

Nir, T. M., Jahanshad, N., Villalon-Reina, J. E., Isaev, D., ZavaliangosPetropulu, A., Zhan, L., ..., Alzheimer's Diseaase Neuroimaginng, I. (2017). Fractional anisotropy derived from the diffusion tensor distribution function boosts power to detect Alzheimer's disease deficits.Magnetic Resonance in Medicine, 78(6), 2322-2333. https://doi.org/10.1002/mrm.26623

Nobis, L., Manohar, S. G., Smith, S. M., Alfaro-Almagro, F., Jenkinson, M., Mackay, C. E., \& Husain, M. (2019). Hippocampal volume across age: Nomograms derived from over 19,700 people in UK Biobank. Neuroimage Clin, 23, 101904. https://doi. org/10.1016/j.nicl.2019.101904

Ozarslan, E., Koay, C. G., Shepherd, T. M., Komlosh, M. E., Irfanoglu, M. O., Pierpaoli, C., \& Basser, P. J. (2013). Mean apparent propagator (MAP) MRI: A novel diffusion imaging method for mapping tissue microstructure. NeuroImage, 78, 16-32. https:// doi.org/10.1016/j.neuroimage.2013.04.016

Pietrasik, W., Cribben, I., Olsen, F., Huang, Y., \& Malykhin, N. V. (2020). Diffusion tensor imaging of the corpus callosum in healthy aging: Investigating higher order polynomial regression modelling. NeuroImage, 213, 116675. https://doi.org/10.1016/j. neuroimage.2020.116675

Pievani, M., Filippini, N., van den Heuvel, M. P., Cappa, S. F., \& Frisoni, G. B. (2014). Brain connectivity in neurodegenerative diseases-from phenotype to proteinopathy. Nature Reviews. Neurology, 10(11), 620-633. https://doi.org/10.1038/nrneurol. 2014.178

Pines, A. R., Cieslak, M., Larsen, B., Baum, G. L., Cook, P. A., Adebimpe, A., ..., Satterthwaite, T. D. (2020). Leveraging multishell diffusion for studies of brain development in youth and young adulthood.Developmental Cognitive Neuroscience, 43, 100788. https://doi.org/10.1016/j.den.2020.100788

Pomponio, R., Erus, G., Habes, M., Doshi, J., Srinivasan, D., Mamourian, E., ..., Davatzikos, C. (2020). Harmonization of large MRI datasets for the analysis of brain imaging patterns throughout the lifespan. Neuroimage, 208, 116450. https://doi. org/10.1016/j.neuroimage.2019.116450

Ritchie, S. J., Cox, S. R., Shen, X., Lombardo, M. V., Reus, L. M., Alloza, C., ..., Deary, I. J. (2018). Sex Differences in the Adult Human Brain: Evidence from 5216 UK Biobank Participants. Cerebral Cortex, 28(8), 2959-2975. https://doi.org/10.1093/ cercor/bhy 109

Royston, P., \& Altman, D. (1994). Regression using fractional polynomials of continuous covariates: Parsimonious parametric modelling. Applied Statistics, 43(3), 429-467.

Salminen, L. E., Wilcox, R. R., Zhu, A. H., Riedel, B. C., Ching, C. R. K., Rashid, F., ..., Jahanshad, N. (2019). Altered Cortical Brain Structure and Increased Risk for Disease Seen Decades After Perinatal Exposure to Maternal Smoking: A Study of 9000 Adults in the UK Biobank. Cereb Cortex, 29(12), 5217-5233. https://doi.org/10.1093/cercor/bhz060

Salminen, L. E., Tubi, M. A., Bright, J., \& Thompson, P. M. (2020). Sex disparities in psychiatric and neurodegenerative disorders: Insights from large-scale neuroimaging. PsyArXiv.

Smith, S. M., Jenkinson, M., Johansen-Berg, H., Rueckert, D., Nichols, T. E., Mackay, C. E., ..., Behrens, T. E. (2006). Tract-based spatial statistics: voxelwise analysis of multi-subject diffusion 
data. Neuroimage, 31(4), 1487-1505. https://doi.org/10.1016/j. neuroimage.2006.02.024

Stejskal, E. O., \& Tanner, J. E. (1965). Spin Diffusion Measurements: Spin Echoes in the Presence of a Time-Dependent Field Gradient. Journal of Chemical Physics, 42(1), 288-+. https:// doi.org/10.1063/1.1695690

Toschi, N., Gisbert, R. A., Passamonti, L., Canals, S., \& De Santis, S. (2020). Multishell diffusion imaging reveals sex-specific trajectories of early white matter degeneration in normal aging. Neurobiology of Aging, 86, 191-200. https://doi.org/10.1016/j. neurobiolaging.2019.11.014

Tseng, W. Y. I., Hsu, Y. C., Chen, C. L., Kang, Y. J., Kao, T. W., Chen, P. Y., \& Waiter, G. D. (2020). Microstructural differences in white matter tracts across middle to late adulthood: A diffusion MRI study on 7167 UK Biobank participants. Neurobiology of Aging, 98, 160-172. https://doi.org/10.1016/j.neurobiola ging.2020.10.006

Wolfers, T., Doan, N. T., Kaufmann, T., Alnaes, D., Moberget, T., Agartz, I., ..., Marquand, A. F. (2018). Mapping the Heterogeneous Phenotype of Schizophrenia and Bipolar Disorder Using Normative Models. JAMA Psychiatry, 75(11), 1146-1155. https:// doi.org/10.1001/jamapsychiatry.2018.2467

Zabihi, M., Oldehinkel, M., Wolfers, T., Frouin, V., Goyard, D., Loth, E., . . Marquand, A. F. (2019). Dissecting the Heterogeneous
Cortical Anatomy of Autism Spectrum Disorder Using Normative Models. Biol Psychiatry Cogn Neurosci Neuroimaging, 4(6), 567-578. https://doi.org/10.1016/j.bpsc.2018.11.013

Zavaliangos-Petropulu, A., Nir, T. M., Thomopoulos, S. I., Reid, R. I., Bernstein, M. A., Borowski, B., ..., Thompson, P. M. (2019). Diffusion MRI Indices and Their Relation to Cognitive Impairment in Brain Aging: The Updated Multi-protocol Approach in ADNI3. Frontiers in Neuroinformatics, 13, 2. https://doi.org/10. 3389/fninf.2019.00002

Zhan, L., Leow, A. D., Zhu, S., Baryshev, M., Toga, A. W., McMahon, K. L., ..., Thompson, P. M. (2009). A novel measure of fractional anisotropy based on the tensor distribution function. Med Image Comput Comput Assist Interv, 12(Pt 1), 845-852. https://doi.org/ 10.1007/978-3-642-04268-3_104

Zhang, H., Schneider, T., Wheeler-Kingshott, C. A., \& Alexander, D. C. (2012). NODDI: Practical in vivo neurite orientation dispersion and density imaging of the human brain. NeuroImage, 61(4), 1000-1016. https://doi.org/10.1016/j.neuroimage.2012.03.072

Publisher's note Springer Nature remains neutral with regard to jurisdictional claims in published maps and institutional affiliations. 